\section{LUPUS SCIENCE\& MEDICINE}

To cite: Olsen NJ, Karp DR. Finding lupus in the ANA haystack. Lupus Science \& Medicine 2020;7:e000384. doi:10.1136/ lupus-2020-000384

Received 18 January 2020 Accepted 20 January 2020

A) Check for updates

(C) Author(s) (or their employer(s)) 2020. Re-use permitted under CC BY-NC. No commercial re-use. See rights and permissions. Published by BMJ.

${ }^{1}$ Division of Medicine, Penn State Milton S Hershey Medical Center, Hershey, Pennsylvania, USA

${ }^{2}$ Department of Internal Medicine, University of Texas Southwestern Medical Center at Dallas, Dallas, Texas, USA

Correspondence to Dr Nancy J Olsen, Medicine, Penn State M.S. Hershey Medical Center, Hershey, PA, United States; nolsen@ pennstatehealth.psu.edu

\title{
Finding lupus in the ANA haystack
}

\author{
Nancy J Olsen (1) , ', David R Karp²
}

\section{ABSTRACT}

Diagnosis of SLE in early stages is challenging due to the heterogeneous nature of presenting symptoms and the poor performance metrics of the screening ANA test. Even the more specific double-stranded DNA autoantibody has relatively low predictive value in early disease. A consequence is delayed referral, with the likelihood that some patients have progression of disease prior to specialist evaluation. Tests that might fill this diagnostic gap are therefore needed. The AVISE Connective Tissue Disease Test that uses a multiplex approach to detect autoantibodies and cell-bound complement products has shown utility in distinguishing SLE from other rheumatological conditions. Whether it might be useful in early disease stages to predict progression is addressed in a recent study by Liang and colleagues, who tested clinic patients who had non-specific findings with the objective of determining whether AVISE could predict onset of SLE. While this test provided more useful prognostic information than other available diagnostics, it had relatively low sensitivity, suggesting that significant numbers of patients with preclinical SLE would be missed by this screening. The need remains for development of diagnostics with robust sensitivity and specificity in early disease that would also deliver prognostic information about risk for SLE. Such tests would have great value as a tool for primary providers to more efficiently triage ANA-positive patients for appropriate specialty evaluation.

Establishing a diagnosis of SLE is currently based on clinical acumen, laboratory testing and adaptive use of criteria that were designed to classify participants for clinical research. ${ }^{12}$ A young woman presenting with a photosensitive malar rash, non-erosive arthritis and typical autoantibodies would not stump an experienced rheumatologist or dermatologist. Complicated diagnostic algorithms in such a scenario are unnecessary. However, the problem of lupus diagnosis extends far beyond this type of presentation on both sides of the equation, the patient and the provider. On the patient side, early detection of SLE remains a challenge. Recent analyses suggest that many patients with SLE present with non-specific symptoms such as fever, myalgia, fatigue or arthritis, rather than more typical lupus findings such as malar rash. ${ }^{34}$ On the provider side of the equation, such patients are most likely to present to primary care physicians or advanced practice providers who are unlikely to be thinking of a rare disease like SLE, and who may not see a need for rheumatological consultation. Delay in getting such a patient to a rheumatologist or lupus specialist is further complicated by the poor performance of the ANA which is the major screening diagnostic. The ANA is extremely sensitive for lupus, but has a high prevalence in the normal population ${ }^{5-7}$ making it too non-specific to be very useful as a screening test. When an ANA-positive patient is referred to a busy rheumatology practice, there is no sense of urgency if other lupus-suggestive symptoms or laboratory tests are not present. In some rheumatology practices, more than $10 \%-15 \%$ of incoming requests for consultation are for evaluation of ANA positivity, so this is not a small problem. Some of these ANA-positive individuals are in an early stage of an evolving lupus syndrome, but identification of those who are at risk is not readily accomplished with available laboratory tests. Therefore, during what can be a long wait time to evaluation, it is likely that some of these ANA-positive patients will have evolution of disease with development of damage at the time of presentation to the lupus specialist. ${ }^{8}$ Even nephropathy has been seen in a significant proportion of early lupus cohorts. $^{9}$

This situation would benefit greatly from improved diagnostic tests with robust sensitivity and specificity and quantitative outputs. These tests would not circumvent the eventual need for specialist evaluation, which requires complex analysis and specialised training. ${ }^{2} 10$ Instead they would be most useful for detecting and triaging patients who are urgently in need of referral to see the specialist. While the presence of antibodies to double-stranded DNA is a long-standing and useful specific biomarker for SLE, the low sensitivity of this test, as well as its low predictive value in individuals with non-specific symptoms, makes it a less than robust screening tool. ${ }^{2}$ Similarly, serum levels of complement proteins C3 and C4 correlate with formation of immune complexes that are central to the pathogenesis of SLE, but while low complement levels are informative about activity in established SLE, early, 
undifferentiated disease may be less commonly associated with depressed complement levels. In a recent study of patients with three American College of Rheumatology (ACR) classification criteria, who were considered to have probable SLE, less than $10 \%$ had hypocomplementemia. ${ }^{11}$

One approach to making better diagnostics is to multiplex tests, combining ones that each have predictive value but that are not correlated with each other. In a Bayesian analysis, the increased probability of disease gained from a positive result in a test associated with the condition can be augmented by a negative result in a test that rules it out. The AVISE Connective Tissue Disease test uses this approach and consists of two parts. The first is a series of autoantibodies whose Bayesian combination produces a numerical result that is akin to a post-test probability. ${ }^{1}$ The second and unique component of this test is measurement of cell-bound complement C4 activation products (CBCAPS). Circulating immune complexes are felt to be central to the pathogenesis of lupus by activating neutrophils and plasmacytoid dendritic cells. ${ }^{12}$ Measurement of complement activation on cell surfaces is a convenient and reproducible method of assessing these soluble immune complexes. A two-tier test strategy is proposed. If a patient has any of the highly specific tests (high titre anti-dsDNA, high titre anti-Sm or extremely high titre cellbound C4d) they are likely to have SLE. While anti-DNA and anti-Sm levels are commonly measured in the evaluation of patients suspected of having lupus and are highly specific biomarkers, they do not identify all patients due to low sensitivity. The addition of cell-bound C4d added approximately $10 \%$ to the diagnostic accuracy, as judged by the area under the receiver operating characteristic curve when compared with the use of hypocomplementemia or anti-DNA titre alone. ${ }^{13}$ However, only $2 \%$ of patients with lupus had extremely elevated erythrocyte C4d and only $14 \%$ of patients with lupus had extremely elevated B cell C4d such that they could be used to signify lupus by themselves, underscoring the importance of autoantibody testing in addition to the CBCAPS. If none of these biomarkers (anti-Sm, anti-DNA or CBCAPS) are highly positive, then an 'index' consisting of various antibody titres and more moderate, but still elevated, levels of cell-bound C4d is calculated. If either the tier 1 or tier 2 test is positive, then the overall test is considered positive. When the test was used in a heterogeneous group of subjects including rheumatoid arthritis (RA), systemic sclerosis, Sjögren's syndrome, inflammatory myositis and healthy controls, the overall sensitivity for lupus was $80 \%$, which is lower than what would be optimal for a screening test. ${ }^{13}$ The test has fair specificity for discriminating lupus from other connective tissue diseases $(70 \%-92 \%)$ and high specificity for discriminating lupus from healthy controls (98\%). It also has been shown to distinguish patients with SLE from those with fibromyalgia (FM) with $100 \%$ specificity, meaning that none of the patients with FM had a positive CBCAPS test result. ${ }^{14}$ Overall, the positive likelihood ratio for the test was 5.6 when trying to differentiate lupus from other rheumatic diseases and
40 when used to distinguish lupus from healthy controls. While these studies suggest that the CBCAPS test characteristics are better than the stand-alone antibody tests in this population, no studies have looked at the costeffectiveness of the CBCAPS test strategy versus standard antibody testing interpreted by an experienced rheumatologist or the potential population health consequences of using CBCAPS as a screening test and missing $20 \%$ of lupus cases.

As noted, most rheumatologists should not find it difficult to tell whether a patient has criteria-defined lupus versus criteria-defined RA/scleroderma. Liang and coauthors ${ }^{15}$ describe the use of the AVISE test to answer a different question, which is whether patients who had undifferentiated findings presenting in the real-world setting of a rheumatology clinic might be assessed for risk of developing SLE. These early-stage patients often have non-specific symptoms, and complement activation may be less prevalent. Predictive tests are in general more difficult to formulate than tests that classify individuals with prevalent disease. One major variable to consider when assessing performance metrics of such a test is the pre-test probability in the study group. The Liang study patients were already in an academic rheumatology clinic, and thus were likely to have a higher pre-test probability of developing SLE or another classifiable connective tissue disease. This is similar to what has been described for preclinical RA in which simply having seen a provider, not necessarily a specialist, and having had a rheumatoid factor (RF) test ordered increases the pre-test probability of RA from the $1 \%$ level in the general population to $17 \%$. This increase was observed even though the reasons for requesting RF were highly variable and often did not include classic symptom findings. ${ }^{16}$ It is clear that the screening ANA, which is also often ordered without clear symptom indicators, does not perform as well for preclinical SLE as does the combination of RF/cyclic citrullinated peptide antibodies in RA, so other tests are needed.

The Liang study examined 117 patients who did not have a baseline diagnosis of SLE. Many of these patients were felt to have 'undifferentiated connective tissue disease'. They found that patients with a positive two-tier multi-antibody/ CBCAPS test were 2.8 times more likely to be given a new diagnosis, most often SLE, after 2 years with a significant accrual of both clinical features of lupus as well as organ damage. These results suggest that the test might be useful not just to detect early SLE but also to identify those with more severe forms of disease. This type of information could be used to initiate therapy such as hydroxychloroquine in earlier stages of SLE, when there is potential to prevent organ-damaging manifestations. ${ }^{17}$ The authors compared the characteristics of the two-tier test in this setting and found it superior to ANA titre, anti-dsDNA and hypocomplementemia in predicting the development of SLE over a 2-year period. Nonetheless, the sensitivity was only $56.5 \%$, suggesting that substantial numbers of patients will be missed if the AVISE test is used for screening. 
Table 1 Candidate diagnostic biomarkers for SLE

\begin{tabular}{lll}
\hline Category & Advantages & Disadvantages \\
\hline Autoantibodies & Present in preclinical SLE & Many overlap with other preclinical disease \\
& Readily measured & states \\
Soluble mediators/ & Present in preclinical SLE & Most are not specific for SLE \\
cytokines & Some (eg, BlyS) may have specificity for SLE & \\
Complement products & Relative specificity for SLE & Not highly prevalent in preclinical disease \\
Gene signatures & Correlation with tissue damage and severity in SLE & \\
& IFN signature present in preclinical disease & Not disease specific; present in RA, \\
& & myositis
\end{tabular}

IFN, interferon; RA, rheumatoid arthritis.

Similar findings were seen in a recent report which documented that high scores on the multianalyte panel that included CBCAPs in patients with three ACR classification criteria was predictive of development of the fourth criterion within 18 months. ${ }^{11}$ Together, these two studies add weight to the concept that SLE is a disease in evolution with evidence of immune system activation (in this case immune complex-driven complement deposition) preceding the accumulation of sufficient clinical and laboratory classification criteria. Whether following patients in either of these studies at regular intervals using standard and available laboratory tests such as urinalysis, complement levels and autoantibodies might also suffice to identify early transformation to SLE classification was not addressed.

Limitations of the Liang study should be recognised. Although the investigators applied the AVISE test in a routine fashion, it was not a prospective trial with set entry criteria. While the types of patients included reflect the reality of referrals to an academic medical practice, it raises the question of whether there was enrichment for patients destined to develop SLE due to referral bias. There is also a question of confirmation bias because the treating physicians were not blinded to the AVISE results. While objective measures (SLICC criteria) were correlated with AVISE, there is no assurance that knowledge of the test did not affect the scoring. This is hinted at by other studies showing that a patient with a positive AVISE test is more likely to be diagnosed with SLE regardless of the number of criteria met, ${ }^{18}$ and that rheumatologists are less likely to diagnose lupus in a patient with a negative AVISE test, regardless of other features. ${ }^{19}$

A major challenge for the development of diagnostics to detect early SLE is to find biomarkers that are relatively disease specific (table 1 ). This is complicated by the fact that SLE along with other many autoimmune diseases in established and preclinical phases share immune features. ${ }^{20}$ ANAs and other autoantibodies are commonly observed in conditions other than those specific for the disease in question. These so-called alternative autoantibodies are especially frequent and broadly represented in SLE and preclinical conditions related to SLE. ${ }^{21}$ Furthermore, more than 180 autoantibodies are associated with
SLE, ${ }^{22}$ which means that the usual clinical panels that include a dozen or less specificities are not providing a wide-angle picture of the autoantibody landscape. Arrays that measure 100 or more autoantibodies simultaneously have been investigated as an approach to developing a more comprehensive autoantibody profile. These arrays have been used to show that subjects with higher SLE risk, including first-degree relatives and those with incomplete lupus (ILE, a condition defined as having one or two criteria for SLE classification in addition to an ANA), have greater IgG autoreactivity than healthy controls. ${ }^{23}$ Another array platform with 200 specificities was developed with a different goal, to rule out SLE in patients suspected of having the disease. The test platform offered quantitative results expressing the likelihood of an SLE diagnosis, which could be useful for sorting out ANApositives in a primary care practice. ${ }^{24} 25$ While this test was capable of distinguishing patients with SLE and those with no SLE, performance in undifferentiated, early or incomplete patients has not been reported.

Other soluble mediators also have potential to detect patients with immune activation in preclinical stages. Some of these may appear even before autoantibodies are measurable. ${ }^{26}$ The B cell stimulator BlyS, for example, shows elevated levels in patients with ILE, though lower than the levels in SLE, suggesting an intermediate and possibly evolving stage of $\mathrm{B}$ cell activation. ${ }^{27}$ Many other circulating cytokines are dysregulated prior to onset of SLE, though not all of these are disease specific. ${ }^{28}$ The signature that reflects activation by Type I interferon, for example, is typical of SLE but is also present in patients with $\mathrm{RA} \cdot{ }^{29} \mathrm{~A}$ disease-specific preclinical cytokine cocktail has not been described.

Complement activation, which is the basis for the AVISE test, is an immune feature that has relatively greater specificity for SLE compared with other autoimmune conditions, such as RA or autoimmune thyroid disorders, that contribute to ANA positivity. Furthermore, activation of complement is associated with organ damage, especially lupus nephritis. ${ }^{30}$ It therefore follows that measurement of CBCAPs has potential to distinguish which ANA-positive individuals are at risk for development of more serious 
disease. A recent report suggests that patients with newly diagnosed SLE have significantly greater prevalence of hypocomplementemia than patients with conditions that mimic SLE, including undifferentiated connective tissue disease. ${ }^{31}$ The patients in this study were collected in clinics staffed by experts in SLE diagnosis and management, which increases the likelihood that disease features would be recognised or measured. Whether AVISE or other tests would be sufficiently informative to risk-stratify ANA-positive patients who are seen in primary practice settings prior to specialty evaluation remains untested.

The field of SLE diagnostics is clearly moving forward at a rapid pace. New test platforms using multiplex approaches are in development for many applications in SLE, including prediction of flare and quantitation of disease activity. In addition to these tests that will help manage patients with established SLE, the need for early risk assessment tools is currently unmet. Most reported studies described here have been carried out in academic rheumatology clinics or specialty practices, rather than at the primary care frontier where the ANA-positive patient, often without any SLE-specific symptoms, is first seen. Bringing testing to this interface will be a necessary step for development of a useful tool to accelerate the diagnosis and treatment of SLE.

Contributors NJO and DRK contributed equally to the writing of this article.

Funding The authors have not declared a specific grant for this research from any funding agency in the public, commercial or not-for-profit sectors.

Competing interests None declared.

Patient consent for publication Not required.

Provenance and peer review Commissioned; internally peer reviewed.

Open access This is an open access article distributed in accordance with the Creative Commons Attribution Non Commercial (CC BY-NC 4.0) license, which permits others to distribute, remix, adapt, build upon this work non-commercially, and license their derivative works on different terms, provided the original work is properly cited, appropriate credit is given, any changes made indicated, and the use is non-commercial. See: http://creativecommons.org/licenses/by-nc/4.0/.

ORCID iD

Nancy J Olsen http://orcid.org/0000-0002-6588-3364

\section{REFERENCES}

1 Ramsey-Goldman R, Li J, Dervieux T, et al. Cell-bound complement activation products in SLE. Lupus Sci Med 2017;4:e000236.

2 Bertsias GK, Pamfil C, Fanouriakis A, et al. Diagnostic criteria for systemic lupus erythematosus: has the time come? Nat Rev Rheumatol 2013;9:687-94.

3 Sebastiani GD, Prevete I, luliano A, et al. The importance of an early diagnosis in systemic lupus erythematosus. Isr Med Assoc J 2016;18:212-5.

4 Leuchten N, Milke B, Winkler-Rohlfing B, et al. Early symptoms of systemic lupus erythematosus (SLE) recalled by 339 SLE patients. Lupus 2018;27:1431-6.

5 Wandstrat AE, Carr-Johnson F, Branch V, et al. Autoantibody profiling to identify individuals at risk for systemic lupus erythematosus. $J$ Autoimmun 2006;27:153-60.

6 Akmatov MK, Röber N, Ahrens W, et al. Anti-nuclear autoantibodies in the general German population: prevalence and lack of association with selected cardiovascular and metabolic disorders-findings of a multicenter population-based study. Arthritis Res Ther 2017;19:127.

7 Pisetsky DS. Antinuclear antibody testing - misunderstood or misbegotten? Nat Rev Rheumatol 2017;13:495-502.
8 Sebastiani GD, Prevete I, luliano A, et al. Early lupus project: oneyear follow-up of an Italian cohort of patients with systemic lupus erythematosus of recent onset. Lupus 2018;27:1479-88.

9 Chen Z, Li M-T, Xu D, et al. Organ damage in patients with incomplete lupus syndromes: from a Chinese academic center. Clin Rheumatol 2015;34:1383-9.

10 Aggarwal R, Ringold S, Khanna D, et al. Distinctions between diagnostic and classification criteria? Arthritis Care Res (Hoboken) 2015;67:891-7.

11 Ramsey-Goldman R, Alexander RV, Massarotti EM, et al. Complement activation in patients with probable systemic lupus erythematosus and ability to predict progression to American College of Rheumatology-classified systemic lupus erythematosus. Arthritis Rheumatol 2020;72:78-88.

12 Rönnblom L, Leonard D. Interferon pathway in SLE: one key to unlocking the mystery of the disease. Lupus Sci Med 2019;6:e000270.

13 Putterman C, Furie R, Ramsey-Goldman R, et al. Cell-bound complement activation products in systemic lupus erythematosus: comparison with anti-double-stranded DNA and standard complement measurements. Lupus Sci Med 2014;1:e000056.

14 Wallace DJ, Silverman SL, Conklin J, et al. Systemic lupus erythematosus and primary fibromyalgia can be distinguished by testing for cell-bound complement activation products. Lupus Sci Med 2016;3:e000127.

15 Liang E, Taylor M, McMahon M. Utility of the AVISE connective tissue disease test in predicting lupus diagnosis and progression. Lupus Sci Med 2020.

16 Silveira IG, Burlingame RW, von Mühlen CA, et al. Anti-CCP antibodies have more diagnostic impact than rheumatoid factor (RF) in a population tested for rf. Clin Rheumatol 2007;26:1883-9.

17 Olsen NJ, James JA, Arriens C, et al. Study of anti-malarials in incomplete lupus erythematosus (SMILE): study protocol for a randomized controlled trial. Trials 2018;19:694.

18 Mossell J, Goldman JA, Barken D, et al. The Avise lupus test and cell-bound complement activation products aid the diagnosis of systemic lupus erythematosus. Open Rheumatol J 2016;10:71-80.

19 Wallace DJ, Alexander RV, O'Malley T, et al. Randomised prospective trial to assess the clinical utility of multianalyte assay panel with complement activation products for the diagnosis of SLE. Lupus Sci Med 2019;6:e000349.

20 Slight-Webb S, Bourn RL, Holers VM, et al. Shared and unique immune alterations in pre-clinical autoimmunity. Curr Opin Immunol 2019;61:60-8.

21 James JA, Chen $\mathrm{H}$, Young KA, et al. Latent autoimmunity across disease-specific boundaries in at-risk first-degree relatives of SLE and RA patients. EBioMedicine 2019;42:76-85.

22 Yaniv G, Twig G, Shor DB-A, et al. A volcanic explosion of autoantibodies in systemic lupus erythematosus: a diversity of 180 different antibodies found in SLE patients. Autoimmun Rev 2015;14:75-9.

23 Li Q-Z, Zhou J, Wandstrat AE, et al. Protein array autoantibody profiles for insights into systemic lupus erythematosus and incomplete lupus syndromes. Clin Exp Immunol 2007;147:60-70.

24 Cohen I, Putterman C, Jordan N, et al. SAT0590 SLE-Keytm Rule-Out Serlogic Test for SLE Using the Immunarray ICHIPTM. Ann Rheum Dis 2015;74:875.1.

25 Putterman C, Wu A, Reiner-Benaim A, et al. SLE-key(®) ruleout serologic test for excluding the diagnosis of systemic lupus erythematosus: developing the ImmunArray iCHIP(囚). J Immunol Methods 2016;429:1-6.

26 Lu R, Munroe ME, Guthridge JM, et al. Dysregulation of innate and adaptive serum mediators precedes systemic lupus erythematosus classification and improves prognostic accuracy of autoantibodies. $J$ Autoimmun 2016;74:182-93.

27 Aberle T, Bourn RL, Munroe ME, et al. Clinical and serologic features in patients with incomplete lupus classification versus systemic lupus erythematosus patients and controls. Arthritis Care Res 2017;69:1780-8.

28 Munroe ME, Young KA, Kamen DL, et al. Discerning risk of disease transition in relatives of systemic lupus erythematosus patients utilizing soluble mediators and clinical features. Arthritis Rheumatol 2017;69:630-42.

29 de Jong TD, Blits M, de Ridder S, et al. Type I interferon response gene expression in established rheumatoid arthritis is not associated with clinical parameters. Arthritis Res Ther 2016;18:290.

30 Buyon J, Furie R, Putterman C, et al. Reduction in erythrocytebound complement activation products and titres of anti-C1q antibodies associate with clinical improvement in systemic lupus erythematosus. Lupus Sci Med 2016;3:e000165. 
31 Mosca M, Costenbader KH, Johnson SR, et al. Brief report: how do patients with newly diagnosed systemic lupus erythematosus present? A multicenter cohort of early systemic lupus erythematosus to inform the development of new classification criteria. Arthritis Rheumatol 2019;71:91-8. 